\title{
VIPMOS-A Novel Buried Injector Structure for EPROM Applications
}

\author{
Rutger C. Wijburg, Gertjan J. Hemink, Student Member, IEEE, Jan Middelhoek, Hans Wallinga, Member, IEEE, \\ and Ton J. Mouthaan, Member, IEEE
}

\begin{abstract}
A buried injector is proposed as a source of electrons for substrate hot electron injection. To enhance the compatibility with VLSI processing, the buried injector is formed by the local overlap of the n-well and p-well of a retrograde twin-well CMOS process. The injector is activated by means of punchthrough. This mechanism allows the realization of a selective injector without increasing the latchup susceptibility. The p-well profile controls the punchthrough voltage. The high injection probability and efficient electron supply mechanism lead to oxide current densities up to $1.0 \mathrm{~A} \cdot \mathrm{cm}^{-2}$. Programming times of $10 \mu \mathrm{s}$ have been measured on nonoptimized cells. The realization of a structure for 5-V-only digital and analog applications is viable. A model of the structure for implementation in a circuit simulator, such as SPICE, is presented.
\end{abstract}

\section{INTRODUCTION}

$\mathrm{A}$ $\mathrm{N}$ increasing demand exists for high-performance EPROM's and $E^{2} P R O M$ 's in digital applications. In the near future, magnetic media may be replaced by $E^{2} P R O M$ 's [1]. However, also in analog and analog-digital applications adjustable components are required. EPROM's can be used in analog CMOS circuits. In this way, it is possible to cancel offsets in differential amplifiers [2]. $E^{2} P R O M$ 's may also find application in adaptive filters or in neural networks [3], [4]. In this paper we will describe a new structure for an EPROM device, which is realized by high-energy ion implantation and may be used in various EPROM applications. The paper focuses on the working principle of the realized structure and its modeling. Furthermore, programming characteristics of nonoptimized memory cells are given, in order to demonstrate its high speed potential. In a following paper, the performance of optimized cells and method of cell selection in a memory matrix will be discussed based on the evaluation of a $16 \mathrm{~K}$ flash-E ${ }^{2}$ PROM [5].

Conventional EPROM's widely use the method of Channel Hot Electron (CHE) injection for programming. Electrons that become hot in the pinchoff region have a small probability of being injected into the gate oxide and flowing to the floating gate. The electrons should gain sufficient kinetic energy in the electrical field parallel to the $\mathrm{Si}-\mathrm{SiO}_{2}$ interface and then they have to be redirected towards the $\mathrm{Si}_{-} \mathrm{SiO}_{2}$ interface [6]. The influence of the drain and gate voltage on the number of hot carriers and the height of the $\mathrm{Si}-\mathrm{SiO}_{2}$ potential barrier lead to conflicting demands [7], [8]. Therefore, high drain and gate voltages are often used as a compromise in practical program-

Manuscript received January 2, 1990; revised June 5, 1990. This work was supported by the Foundation for Fundamental Research on Matter (FOM) and the Netherlands Technology Foundation (STW). The review of this paper was arranged by Associate Editor B. Ricco.

R. C. Wijburg, G. J. Hamink, H. Wallinga, and T. J. Mouthaan are with the Faculty of Electrical Engineering, University of Twente, P.O. Box 217, 7500 AE Enschede, The Netherlands.

J. Middelhoek, deceased, was with the Faculty of Electrical Engineering, University of Twente, 7500 AE Enschede, The Netherlands.

IEEE Log Number 9040178. ming. As a result, drain-side CHE injection EPROM's have a low injection probability. They generally do not allow 5-V-only operation, although for some drain-side CHE injection EPROM's with submicrometer channel length the programming drain voltage can be reduced to $5 \mathrm{~V}$ [9]. The source-side CHE injection technique can be used, in order to overcome these disadvantages [7]. However, as in the case of a drain-side CHE injection EPROM, the injection of hot electrons is limited to a very small region. As the charge injection is the driving mechanism for oxide breakdown [10], the lifetime and the number of programming cycles of such device for $E^{2}$ PROM applications are restricted.

An alternative method to the CHE injection is the Substrate Hot Electron (SHE) injection, which is shown in Fig. 1. The source of electrons is the substrate. The SHE injection has several advantages. Electrons that are accelerated in the depletion layer underneath the gate and become hot are directed towards the $\mathrm{Si}-\mathrm{SiO}_{2}$ interface. The injection probability can be increased by raising the gate voltage, which lowers the $\mathrm{Si}-\mathrm{SiO}_{2}$ potential barrier [11]-[13]. In contrast to the conventional CHE injection, this can be done without decreasing the number of hot electrons. The SHE injection technique can be applied in a $5-\mathrm{V}$-only EPROM, because the only higher voltage needed on chip is connected to the gate terminal of high impedance and can be generated on chip by charge pumping techniques [14]. The injection of electrons can take place over nearly the whole active gate area. In the case of equal injection current the SHE injection offers a lower oxide current density than the CHE injection. Because the oxide is stressed less locally, a higher reliability of the SHE injection EPROM may be expected compared to the CHE injection EPROM. In addition, both normal CMOS and EPROM devices can use the same advanced source and drain structures in order to suppress unwanted shortchannel effects [15].

The major problem associated with the conventional SHE injection is the method for generation of electrons in the substrate. Photogeneration and avalanche multiplication have been used. Verwey et al. [16] employed a forward-biased n-type substrate with a p-type epilayer. Eitan et al. [17] used a forwardbiased p-n junction, which was situated closely to the EPROM device. Such a forward-biased $\mathrm{p}-\mathrm{n}$ junction has low injection efficiency, because only a small part of the injected carriers $\left(10^{-3}\right.$ to $\left.10^{-2}[18]\right)$ enters the depletion layer under the gate. The majority of these carriers either recombines in the substrate or are directly collected by the source and drain without any probability of surmounting the $\mathrm{Si}-\mathrm{SiO}_{2}$ potential barrier. Although the last technique has been implemented in a memory array [19], none of the above-mentioned techniques seems to be a viable way for integration in CMOS circuits because of the danger of latchup. 


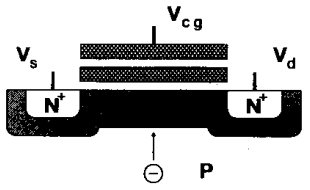

Fig. 1. A conventional substrate hot electron injection EPROM structure. The acceleration of electrons occurs in the depletion layer (hatched) perpendicularly to the monosilicon surface.

In spite of the promising programming method for EPROM's, the SHE injection was not attractive, because it lacked an efficient source of electrons. This problem has been overcome in the so-called VIPMOS structure [19]. The acronym VIPMOS stands for Vertical Injection Punchthrough-based MOS. This structure results in a local buried injector as the source of electrons. The basic device principle is explained in Section II. Section III deals with the device fabrication. Simulations emphasize some of the advantages of this device. Furthermore, a model for implementation in a circuit simulator is presented in Section IV. Experimental results are given in Section V and Section VI summarizes the conclusions.

\section{Basic Principle}

Fig. 2 visualizes the VIPMOS structure. The VIPMOS structure has an additional n-type doped area underneath the gate. This n-type area is the buried injector and acts as the source of electrons. Apart from the injector, the structure can be similar to the conventional SHE injection EPROM, as shown in Fig. 1 .

In the programming mode, the injector is grounded. A high voltage $V_{c g}$ is applied to the control gate. Fig. 2(a) shows the VIPMOS EPROM in the case of connecting both the source and drain to a voltage $V_{d i}$. The substrate area between the MOS stack and injector is not completely depleted. Increasing the voltages on the source and drain will extend the depletion layer underneath the gate into the direction of the injector. At a certain voltage $V_{\text {dep }}$, the depletion layer will touch the depletion layer at the injector side. Further increase of the voltage leads to punchthrough (Fig. 2(b)). The punchthrough voltage $V_{p t}$ is defined as the voltage on the source and drain, when the injector current increases and starts to deviate from the junction saturation current. In the punchthrough mode the injector emits electrons into the depletion layer under the floating gate. These electrons will be accelerated in the electrical field. Some of them will become hot and gain sufficient energy to surmount the $\mathrm{Si}-$ $\mathrm{SiO}_{2}$ potential barrier. The injection mechanism is very efficient, because all electrons emitted by the injector are accelerated in the direction towards the $\mathrm{Si}-\mathrm{SiO}_{2}$ interface and have a chance of being collected by the floating gate. This will be illustrated in the next section. Utilization of the punchthrough mechanism for application in bipolar ROM's has already been reported by Lohstroh et al. [20]. Mouthaan et al. [21] used the punchthrough mechanism in a dynamic RAM cell. In the device, presented in this paper, it is combined with the generation of hot electrons for nonvolatile memories. Actually, the local buried injector, that is activated by means of punchthrough, combines the previously described advantages of the SHE injection with an efficient source of electrons.

Fig. 3 shows the energy band diagram of the VIPMOS structure under programming and read condition. The device can be characterized by two potential barriers $\phi_{b}$ and $\phi_{f .} \phi_{f}$ is the po-

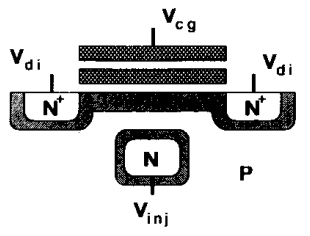

(a)

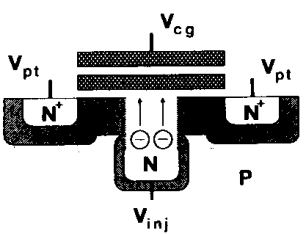

(b)

Fig. 2. The VIPMOS structure with the local buried injector. At $V_{s}$ and $V_{d}$ equal $V_{d i}$ the punchthrough condition is not satisfied (a). Both depletion layers just touch for the case that $V_{s}$ and $V_{d}$ equal $V_{\text {dep }}$. At a voltage $V_{\mathrm{pt}}$, punchthrough occurs and the injector emits electrons into the depletion layer underneath the gate $(\mathrm{b})$.

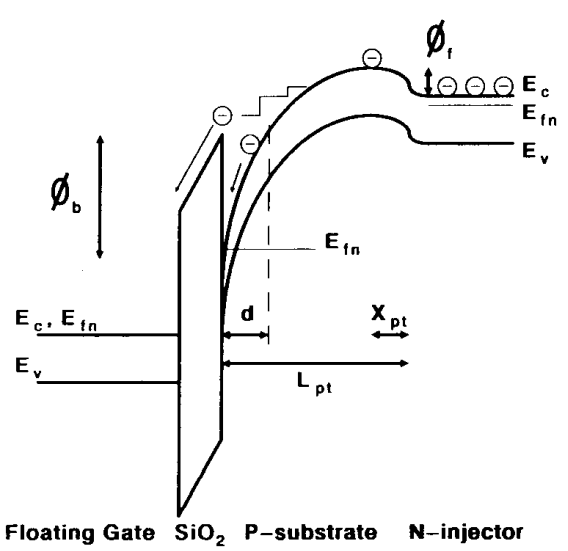

(a)

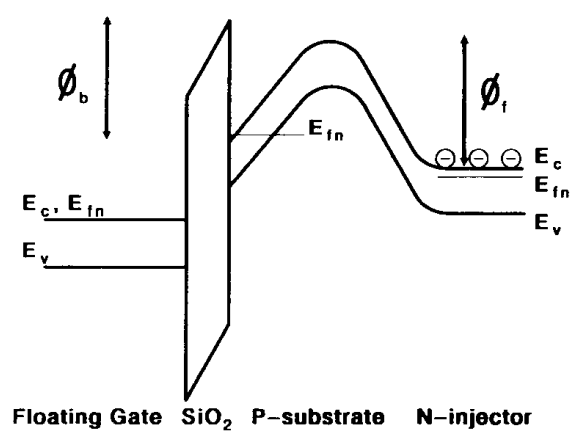

(b)

Fig. 3. The energy band diagram of the VIPMOS structure under programming (a) and read (b) condition. The device is characterized by two potential barriers $\phi_{f}$ and $\phi_{b}$. $\phi_{f}$ is the barrier between the injector and the substrate area above the injector, whereas $\phi_{b}$ is the $\mathrm{Si}-\mathrm{SiO}_{2}$ barrier. 
tential barrier between the injector and the substrate area above the injector, whereas $\phi_{b}$ is the $\mathrm{Si}-\mathrm{SiO}_{2}$ potential barrier. In the programming mode, $\phi_{f}$ is decreased when the source and drain voltage exceed $V_{\text {dep }}$. Electrons will diffuse over the lowered barrier (Fig. 3(a)) and are accelerated towards the $\mathrm{Si}-\mathrm{SiO}_{2}$ interface. Some of them may surmount the $\mathrm{Si}-\mathrm{SiO}_{2}$ barrier, the majority will be drained by either the source or drain. Fig. 3(b) shows the energy band diagram in the read mode. The injector potential is raised (e.g., it is connected to the drain voltage). Thus the barrier $\phi_{f}$ is increased, thereby preventing the emission of electrons from the injector.

In the punchthrough mode, the injector current can be written as

$$
I_{\mathrm{inj}}=I_{0} \exp \left(\frac{q \Delta \phi}{k T}\right)
$$

with

$$
\Delta \phi=\phi_{j 0}-\phi_{f} .
$$

$\phi_{f}$ is the height of the potential barrier as indicated in Fig. 3(a) and $\phi_{j 0}$ is the built-in potential of the junction between the injector and the substrate area above the injector. The dependence of $\Delta \phi$ on the channel potential $\phi_{\mathrm{ch}}$ can be written as [22], [23]

$$
\Delta \phi=\frac{\phi_{\mathrm{ch}}-\phi_{\mathrm{dep}}}{n}
$$

where $\phi_{\text {dep }}$ is the channel potential, when the depletion layer under the gate just touches the depletion layer at the injector side. The nonideality factor $n$ denotes the fraction of the increasing channel potential that is available to forward bias the junction between the injector and the substrate area above the injector. Our devices show typical nonideality factors of 3 to 4 . Using (1) and (3), the punchthrough current may be written as

$$
I_{\text {inj }}=I_{0} \exp \left(\frac{q\left(\phi_{\text {ch }}-\phi_{\text {dep }}\right)}{n k T}\right) \text {. }
$$

In fact, the nonideality factor $n$ is not a constant, but it gradually increases with increasing $\left(\phi_{\mathrm{ch}}-\phi_{\mathrm{dep}}\right)$. Assuming a highly doped injector and a homogeneously doped substrate area above the injector, a more precise expression for $\Delta \phi$ is given by [22], [23]

$$
\Delta \phi=\frac{x_{\mathrm{pt}}}{L_{\mathrm{pt}}}\left(\phi_{\mathrm{ch}}-\phi_{\mathrm{dep}}\right)\left(1+\frac{x_{\mathrm{pt}}}{L_{\mathrm{pt}}} \frac{\phi_{\mathrm{ch}}-\phi_{\mathrm{dep}}}{4 \phi_{j 0}}\right)
$$

with $x_{\mathrm{pt}}$ is the distance from the potential minimum, when $\phi_{\mathrm{ch}}$ $=\phi_{\text {dep }}$, to the injector and $L_{\mathrm{pt}}$ the distance from the $\mathrm{Si}-\mathrm{SiO}_{2}$ interface to the injector, as indicated in Fig. 3(a).

At high currents, $I_{\text {inj }}$ will become space-charge-limited and it can no longer be described by an exponential behavior as in (4). For the derivation of the injector current it is assumed that the injector is grounded. For the case it is not grounded or it has a considerable resistance, $\phi_{\mathrm{ch}}$ has to be replaced by the potential difference across the punchthrough structure.

Hot electron injection is often modeled by the Lucky Electron Model (LEM) [11]-[13]. This model was originated by Shockley [24] and later refined by Verwey et al. [11], [12] and Ning et al. [13]. Following this model, the probability for an electron of being injected over the potential barrier $\phi_{b}$ is given by

$$
P_{\mathrm{inj}}=A \exp \left(-\frac{d}{\lambda}\right)
$$

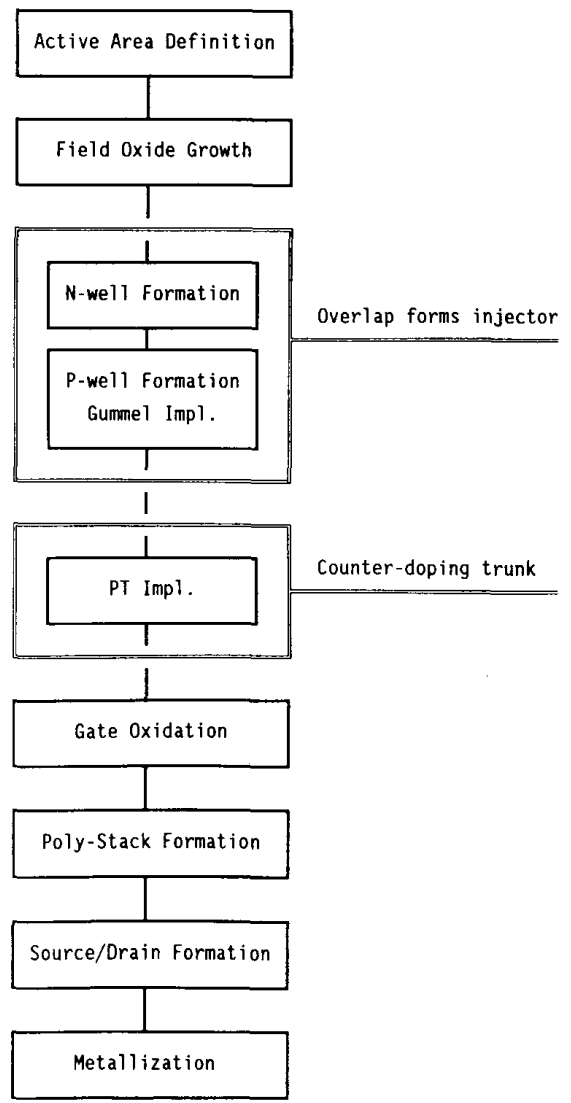

Fig. 4. Process flow forming the VIPMOS structure.

where $A$ is a fitting constant. $d$ is the minimum distance which an electron has to travel without suffering any collisions, in order to acquire an energy equal to the $\mathrm{Si}-\mathrm{SiO}_{2}$ barrier $\phi_{b}$, as indicated in Fig. 3(a). $\lambda$ is the scattering mean free path of an electron. Using the solution of the Poisson equation, the following expression for $d$ can be derived, assuming a substrate area above the injector that is homogeneously doped to a concentration of $N_{a}$

$$
d=\sqrt{\frac{2 q \epsilon_{\mathrm{si}} \phi_{\mathrm{ch}}}{N_{a}}}\left(1-\sqrt{1-\frac{\phi_{b}}{\phi_{\mathrm{ch}}}}\right), \quad \phi_{\mathrm{ch}} \leq \phi_{\mathrm{dep}}
$$

where $q$ is the electronic charge and $\epsilon_{\mathrm{si}}$ is permittivity of silicon. The height of the barrier $\phi_{b}$ is affected by the electrical field in the gate oxide. Ning derived an expression for $\phi_{b}$, which incorporates the image force barrier lowering term and a "tunneling barrier lowering"' term [13]

$$
\phi_{b}=3.2-\beta E_{\mathrm{ox}}^{1 / 2}-\alpha E_{\mathrm{ox}}^{2 / 3}
$$

with $E_{\mathrm{ox}}$ is the electrical field in the gate oxide. $\alpha$ and $\beta$ are constants. $E_{\text {ox }}$ is calculated by

$$
E_{\mathrm{ox}}=\frac{V_{\mathrm{flg}}-V_{\mathrm{fb}}-\phi_{\mathrm{ch}}}{T_{\mathrm{ox}}}
$$

with $V_{\mathrm{fg}}$ is the floating gate voltage, $V_{\mathrm{fb}}$ the flatband voltage, and $T_{\mathrm{ox}}$ the gate-oxide thickness. 


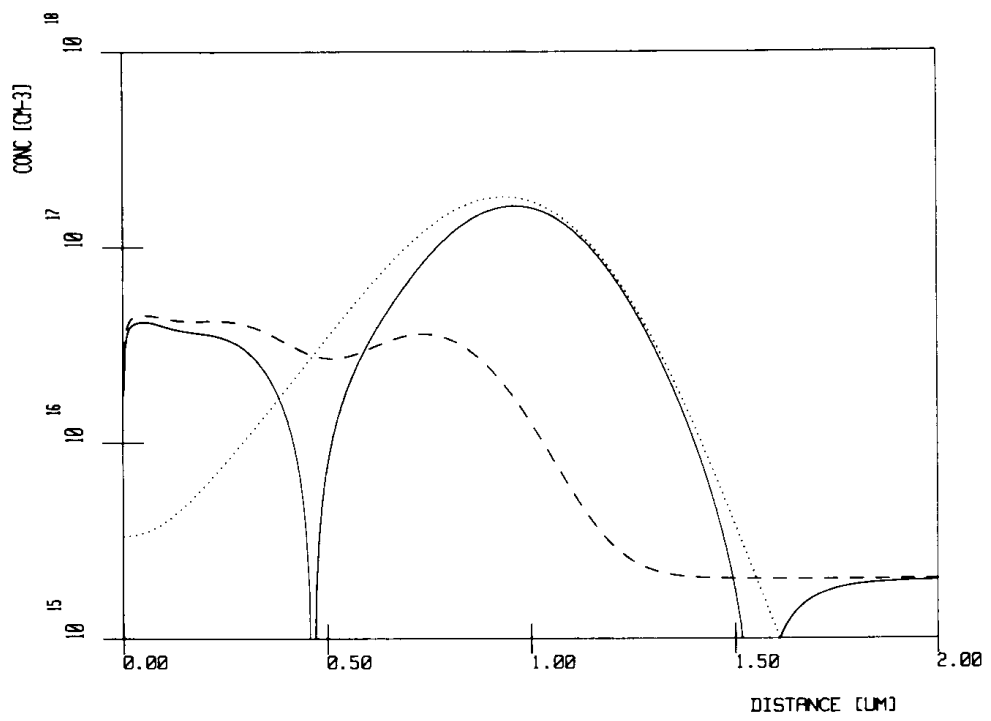

Fig. 5. The 1-D-doping profile of the buried injector as simulated with SUPREM III. The solid line represents the net doping concentration, the dotted line the phosphorus concentration, and the dashed line the boron concentration.

Using the above-mentioned equations, the current flowing to the floating gate can be calculated as

$$
I_{\mathrm{flg}}=P_{\text {inj }} \times I_{\text {inj }}
$$

thereby combining the LEM with the punchthrough model.

\section{Process Description and Simulation}

The process simplicity makes the application of VIPMOS structures attractive for custom CMOS applications. The buried injector structure can easily be realized by high-energy ion implantation. In this way, the Gummel number of the substrate area above the injector can be well-controlled. This is important, because the punchthrough voltage is sensitive to the Gummel number. To create a local injector, the conventional buried layer does not seem to be favorable. The possible spread in the thickness and doping concentration of the epitaxial layer result in a nonreproducible Gummel number of the region above the injector.

Essentially, the buried injector is formed just by an overlap of the retrograde n-well and p-well of our high-energy ion-implanted CMOS process [25]. The process flow is given in Fig. 4. The Gummel number of the substrate area above the injector is given by

$$
Q=\int_{0}^{L_{p 1}-x_{p t}}\left(N_{a}(x)-N_{d}(x)\right) d x .
$$

$N_{a}(x)$ and $N_{d}(x)$ are determined by the p-well and n-well profile, respectively. The wells are implanted immediately after the field oxidation. The retrograde n-well, in which the normal PMOS devices are formed, is made by a single implantation step of $1-\mathrm{MeV}$ phosphorus ions and a dose of $1 \times 10^{13} \mathrm{~cm}^{-2}$. The retrograde $\mathrm{p}$-well is formed by a boron implantation with an energy of $350 \mathrm{keV}$ and a dose of $1.5 \times 10^{12} \mathrm{~cm}^{-2}$. The retrograde p-well implantation determines the threshold voltage of the parasitic field-oxide NMOS. This implantation is fully covered by the overlapping n-well implantation at the injector
TABLE I

Different Gummel and Threshold Adjustment Implantations that Have BeEn Used to Vary the p-

Well Profile, in Order to INVestigate its Influence on the Punchthrough Voltage, Injection PROBABILITY, AND NONIDEALITY FACTOR $n$

\begin{tabular}{ccc}
\hline & \multicolumn{2}{c}{ Dose $\left(\mathrm{cm}^{-2}\right) /$ Energy $(\mathrm{keV})$} \\
\cline { 2 - 3 } Device & $\begin{array}{c}\text { Gummel } \\
\text { Implantation }\end{array}$ & $\begin{array}{c}\text { Threshold } \\
\text { Adjustment }\end{array}$ \\
\hline A & $1.5 \times 10^{12} / 210$ & - \\
& $1.5 \times 10^{12} / 70$ & - \\
B & $1.0 \times 10^{12} / 210$ & - \\
& $1.5 \times 10^{12} / 70$ & $5.0 \times 10^{11} / 10$ \\
C & $2.0 \times 10^{12} / 150$ & $2.0 \times 10^{11} / 40$ \\
D & $1.5 \times 10^{12} / 110$ & $5.0 \times 10^{11} / 10$ \\
& & $2.0 \times 10^{11} / 40$ \\
\hline
\end{tabular}

side. An additional boron implantation with an energy of 110 $\mathrm{keV}$ and a dose of $1.5 \times 10^{12} \mathrm{~cm}^{-2}$ is done through the same mask. This implantation, further referred to as Gummel implantation, is necessary to suppress the front flank of the retrograde n-well profile. The Gummel implantation ensures a reproducible Gummel number of the substrate area above the injector. The p-well loses its retrograde character due to the Gummel implantation. The threshold voltage of the normal NMOS devices is determined by two boron implantations. The first boron implantation with an energy of $40 \mathrm{keV}$ and a dose of $2 \times 10^{11} \mathrm{~cm}^{-2}$ is performed during the $\mathrm{p}$-well formation. The second boron implantation, that also covers the threshold voltage adjustment of the normal PMOS devices, is a blanket implantation with an energy of $10 \mathrm{keV}$ to a dose of $5 \times 10^{11} \mathrm{~cm}^{-2}$ through the gate oxide with a thickness of $25 \mathrm{~nm}$. Obviously, these boron implantations also affect the Gummel number of the substrate area above the injector. The doping profile of the bur- 


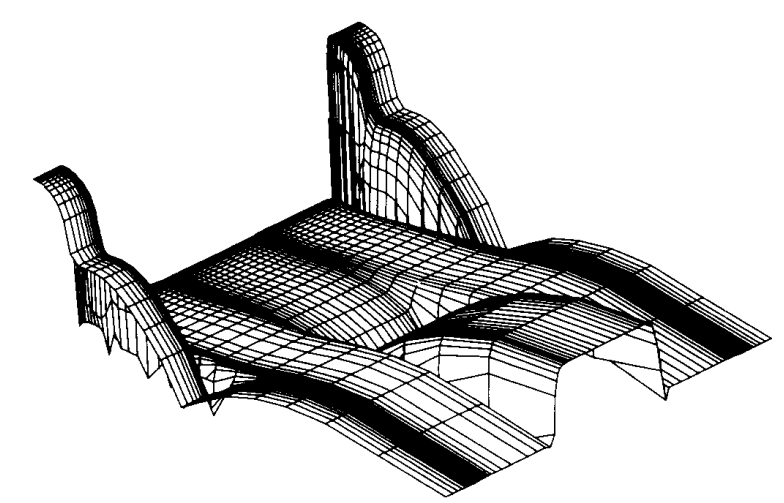

Fig. 6. 2-D-doping profile of the VIPMOS structure, which is used for the device simulations.

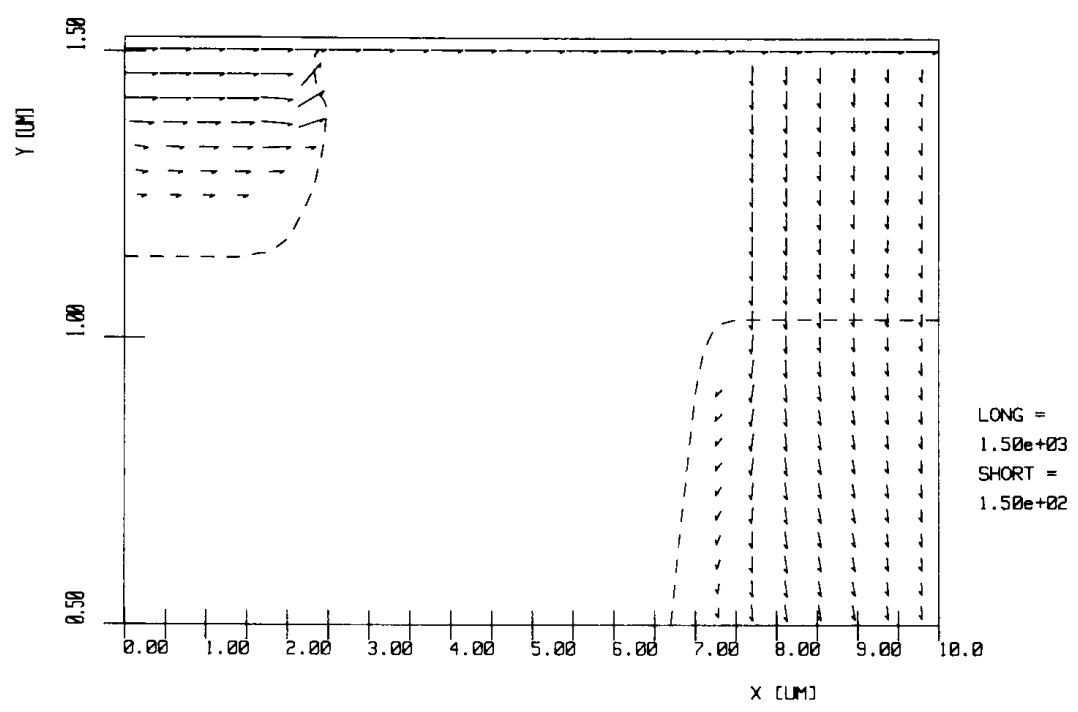

Fig. 7. A vector plot of the electron current density in programming mode. The dashed lines denote the junctions. The spacing between the injector and source is $5 \mu \mathrm{m}$. The injector is grounded $V_{s}=5 \mathrm{~V}$ and $V_{\mathrm{fgg}}=10 \mathrm{~V}$. The injection of electrons from the injector only occurs in the direction of the floating gate.

ied injector, as simulated with SUPREM III [26], is shown in Fig. 5. The junction depth, resulting from the p-well and n-well overlap (device D in Table I), is about $0.5 \mu \mathrm{m}$.

In principle, the buried injector can be realized without any extra masking step. However, in the case of the formation of high-energy ion-implanted buried layers an n-type trunk from the buried layer up to the surface arises at the mask edge [27]. The trunk implies an electrical connection between the buried layer and the inversion layer, preventing the VIPMOS structure from operation. In order to tackle this problem, an additional PT-masked (Preventing Trunk) implantation, $150-\mathrm{keV}$ boron ions up to a dose of $5 \times 10^{12} \mathrm{~cm}^{-2}$, is carried out at the edges of the injector. Unfortunately, the PT mask increases the device dimensions at this moment.

The Gummel implantation will scarcely affect the threshold voltage of the normal NMOS devices, because it is a deeper implantation. On the other hand it will considerably increase the bulk factor, especially when higher doses are used in order to obtain a high punchthrough voltage. Also the PT implantation will have a profound effect on the bulk factor.

It is interesting to investigate the influence of the Gummel number of the substrate area above the injector on the injection probability. Generally, a high Gummel number results in a high punchthrough voltage and high injection probability, whereas a lower Gummel number will give a lower punchthrough voltage and lower injection probability [28]. In addition to the Gummel number of the region above the injector, the resulting shape of the profile of the p-well influences the injection probability. A high boron peak near the $\mathrm{Si}-\mathrm{SiO}_{2}$ surface locally enhances the electrical field and leads to a higher injection probability. The shape of the p-well profile also influences the nonideality factor $n$. A deeper Gummel implantation will shift the position of the potential barrier $\phi_{f}$ into the direction of the injector, thereby increasing the nonideality factor $n$. In order to investigate these aspects, the Gummel implantation and the threshold adjustment implantations were varied. This resulted in four devices A to D 


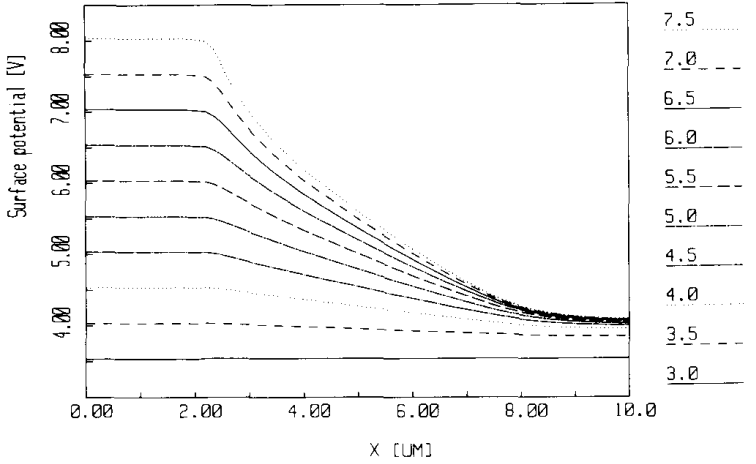

Fig. 8. The potential along the $\mathrm{Si}-\mathrm{SiO}_{2}$ interface at different source voltages. The source junction is situated at $x=2 \mu \mathrm{m}$ and the injector junction at $x=7 \mu \mathrm{m}$. At a certain voltage, the channel potential is no longer constant along the whole injector area.

as listed in Table I. The retrograde p-well and n-well implantations were identical for all devices. Section $\mathrm{V}$ will deal with the measurements resulting from these devices.

Fig. 6 shows the 2-D doping profile of the VIPMOS structure. This profile is used for device simulation with TRENDY, a 2-D device simulator [29]. In the programming mode the device behavior is symmetrical. Therefore, only one half of the structure is simulated in order to save computation time. A vector plot of the electron current density under programming condition is shown in Fig. 7. The electron current density from injector towards the channel easily exceeds $200 \mathrm{~A} \cdot \mathrm{cm}^{-2}$ in the programming mode. The electron current density is not constant along the whole injector area. The local injector current density as well as the local injection probability is determined by the channel potential above the injector area. This channel potential itself depends on the amount of charge, which can be derived from the channel by the source and drain. This effect is emphasized in Fig. 8. It displays the channel potential along the surface from source to injector at different source voltages. Notice that at a certain source voltage, the channel potential apparently has no longer a constant value along the whole channel. This restricts the applicability of the LEM, which will be explained in the following section.

As can be seen from Fig. 7, the injection of electrons occurs in vertical direction. Moreover, the injection does not occur in lateral direction, where it might trigger other structures. Holes, generated by impact ionization in the depletion layer under the floating gate, contribute to the substrate current. This substrate current may cause a parasitic bipolar action [30]. The influence of the substrate current is suppressed by the use of a heavily doped p-type substrate [25]. Therefore, the VIPMOS structure seems to be suitable for application in VLSI circuits without the danger of latchup.

\section{VIPMOS MODEL}

Fig. 9 shows a typical plot of the measured injector current as a function of the drain/source to bulk voltage $\left(V_{\mathrm{dsb}}\right)$ at different "floating" gate voltages $\left(V_{\mathrm{fg}}\right)$. At drain/source voltages only slightly higher than the punchthrough voltage, the current has a logarithmic behavior and can be well described by (1) and (5). The injector current is independent of $V_{\mathrm{flg}}$. The potential in the channel above the injector region is constant and fixed to a

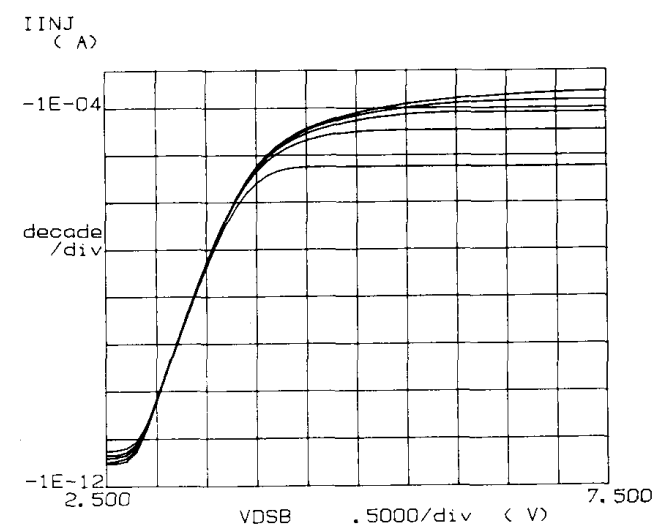

Fig. 9. Typical curves of a VIPMOS device. $V_{\mathrm{fg}}$ varies from 7.5 to 11.5 $\mathrm{V}$ in steps of $1 \mathrm{~V}$. At low $V_{\mathrm{dsb}}$ the current is independent of $V_{\mathrm{fig}}$. At higher $V_{\mathrm{dsb}}$ the MOS action dominates the device.

value of about

$$
\phi_{\mathrm{ch}}=V_{\mathrm{dsb}}+2\left|\phi_{p}\right|
$$

with $\phi_{p}$ is the substrate Fermi potential. In this case the injector current can be modeled with the help of the previous equations. For the "floating" gate current the LEM can be used. At higher $V_{\mathrm{dsb}}$, the current is dominated by the MOS action. The drain/ source to bulk voltage, at which this applies, is further referred to as $V_{\mathrm{tr}}$. For $V_{\mathrm{dsb}}$ greater than $V_{\mathrm{tr}}$, the potential in the channel will gradually increase from the middle of the injector to the source and drain. This implies that the injection will not be homogeneous, and moreover the injection probability varies over the injector area. Electrons that are injected closer to either the source or drain have a greater probability of surmounting the $\mathrm{Si}-\mathrm{SiO}_{2}$ potential barrier. Thus the injection probability will not only be dependent on $V_{\mathrm{flg}}$, but also on $V_{\mathrm{dsb}}$. This phenomenon is more pronounced for devices with a low Gummel number of the substrate area above the injector. A more precise expression for the "floating" gate current is

$$
I_{\mathrm{fg}}=\int_{x=a}^{x=b} P_{\mathrm{inj}}(x) I_{\mathrm{inj}}(x) d x
$$

where $a$ and $b$ are the boundaries of the injection area. This effect makes a use of the LEM for SPICE modeling inconvenient, because a precise knowledge of the channel potential is required. Therefore, an empirical relation for the injection probability is introduced

$$
P_{\text {inj }}=P_{0}+P_{k} I_{\text {inj }}\left(V_{\text {flg }}-V_{P 0}\right) .
$$

In (14), $P_{0}$ is the injection probability at an arbitrary "floating", gate voltage $V_{P 0}$ and $P_{k}\left(\mathrm{~A}^{-1} \mathrm{~V}^{-1}\right)$ is a fitting constant.

In Fig. 10 the model for the VIPMOS structure is given. This model has been implemented in SPICE. The punchthrough current source is modeled with the help of (1) and (5). We have used the SPICE level3 MOS model [31] for the MOS behavior. The floating gate current is included in the model, using (10) and (14). $R_{\mathrm{inj}}$ is the resistance of the injector. It should necessarily be incorporated in the model, because the injector sheet resistance is $1000 \Omega / \square$. The parameters for the model are obtained from the parameter extraction program PROMEA [32] and will be discussed in the next section. 


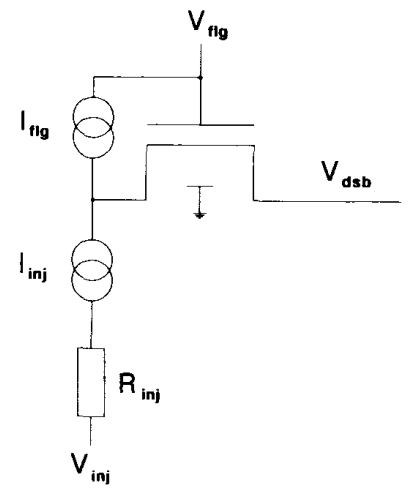

Fig. 10. Model for the VIPMOS structure that has been implemented in SPICE.

\section{MEASUREMENTS AND Discussion}

We have fabricated several VIPMOS structures with different punchthrough voltages by varying the doping profile of the p-well. Fig. 11(a) shows the measured injector and "floating", gate current of device $\mathrm{A}$ as a function of the drain/source to bulk voltage $V_{\mathrm{dsb}}$. The "floating" gate voltage $V_{\mathrm{flg}}$ is $15 \mathrm{~V}$. The punchthrough voltage of this device is $6.85 \mathrm{~V}$ and $V_{\mathrm{tr}}$ is $8.0 \mathrm{~V}$. The injection probability at the point indicated by the marker is nearly $1 \times 10^{-3}$. The injector area of this device equals 12.5 $\mu \mathrm{m}^{2}$. It can be calculated that the electron current density in the gate oxide exceeds $0.4 \mathrm{~A} \cdot \mathrm{cm}^{-2}$. At higher values of $V_{\mathrm{dsb}}$ and $V_{\text {ffg }}$ oxide current densities of $1 \mathrm{~A} \cdot \mathrm{cm}^{-2}$ are measured. These high values are acquired as a result of a high injection probability accompanied by an efficient electron injection mechanism. In Fig. 11(b) the injector current and "floating" gate current of the same device are plotted as a function of $V_{\mathrm{fg}} . V_{\mathrm{dsb}}$ is $9 \mathrm{~V}$. It is clearly demonstrated that below a certain value of $V_{\mathrm{flg}}$ the injector does not emit electrons, because the punchthrough condition cannot be satisfied at low $V_{\text {flg }}$. Consequently, the injection automatically stops in such an EPROM, when a sufficient amount of charge carriers is accumulated on the floating gate.

The high oxide electron current densities may result in estimated programming times in the microsecond range for optimized structures. These expectations are based upon measurement results shown in Fig. 12. This figure displays the programming characteristics of devices A and D. Device A has a high injection probability and device $\mathrm{D}$ can be programmed with a low drain voltage of $5 \mathrm{~V}$. These devices are not optimized. The total floating gate capacitance $C_{\text {tot }}$ is approximately $0.8 \mathrm{pF}$, the coupling ratio has a value of 0.6 , and the injector area is $12.5 \mu \mathrm{m}^{2}$. Notwithstanding its considerable $C_{\text {tot }}$, device A reveals a threshold voltage shift of about $3 \mathrm{~V}$ within $10 \mu \mathrm{s}$. Optimized devices with the same injector area can have a $C_{\text {tot }}$ less than $0.1 \mathrm{pF}$. This will result in programming times which are approximately one order of magnitude shorter than the times shown in Fig. 12. The relatively high control gate voltages can be reduced by increasing the coupling ratio and decreasing the threshold voltage shift. Currently, no optimized cell structures are available. However, a $5-\mathrm{V}$-only $16 \mathrm{~K}$ flash $\mathrm{E}^{2} \mathrm{PROM}$ with optimized VIPMOS structures is under development [5].

For analog applications, such as neural networks [4], a VIPMOS structure with a lower punchthrough voltage is desirable for supply voltage considerations. Thus a tradeoff should be

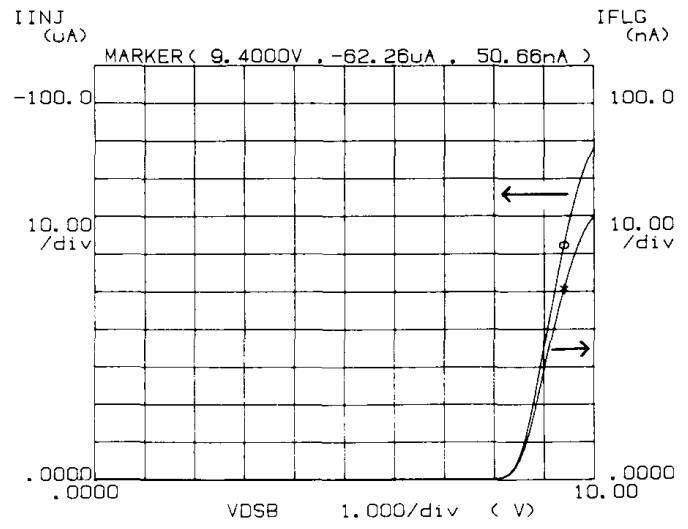

(a)

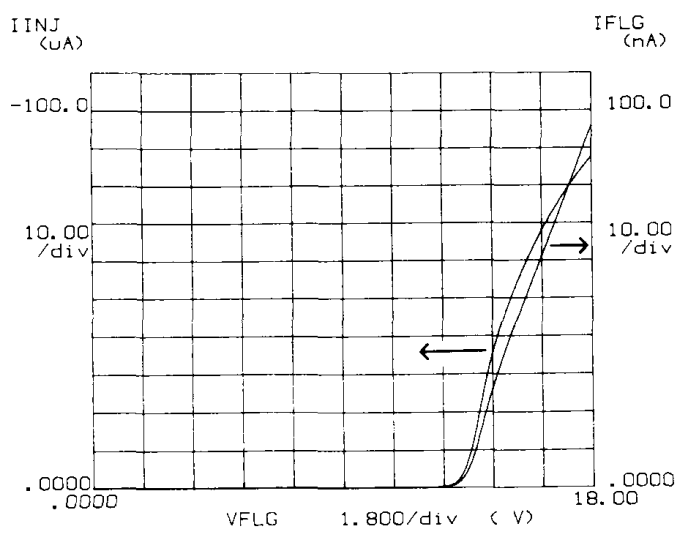

(b)

Fig. 11. The measured injector (left axis) and "floating" gate current (right axis) of a device $\mathrm{A}$ as a function of the drain/source to bulk voltage ( $\left.V_{\mathrm{dsb}}\right)$ with the "floating" gate voltage $\left(V_{\mathrm{fg}}\right)$ is $15 \mathrm{~V}$ (a) and as a function of $V_{\mathrm{flg}}$ with $V_{d s b}$ is $9 \mathrm{~V}(\mathrm{~b})$.

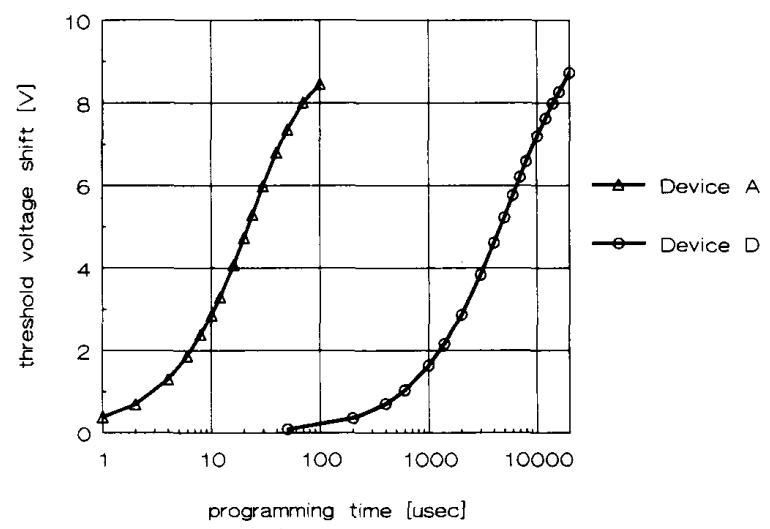

Fig. 12. Measured programming times of the nonoptimized devices $A$ and D. Programming conditions for device $\mathrm{A}$ are: $V_{\mathrm{dsb}}=10 \mathrm{~V}$ and $V_{\mathrm{cg}}=25$ $\mathrm{V}$. For device $\mathrm{D}$ the conditions are: $V_{\mathrm{dsb}}=5 \mathrm{~V}$ and $V_{\mathrm{cg}}=20 \mathrm{~V}$.

made between the injection probability and the supply voltage. Fig. 13 shows the characteristics of device D. The punchthrough voltage $V_{\mathrm{pt}}$ of device $\mathrm{D}$ is $2.75 \mathrm{~V}$ and $V_{\mathrm{tr}}$ is $3.8 \mathrm{~V}$. The squares in the figure represent the data that have been stored 


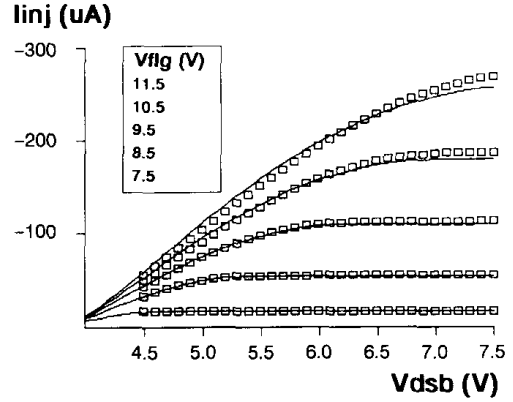

(a)

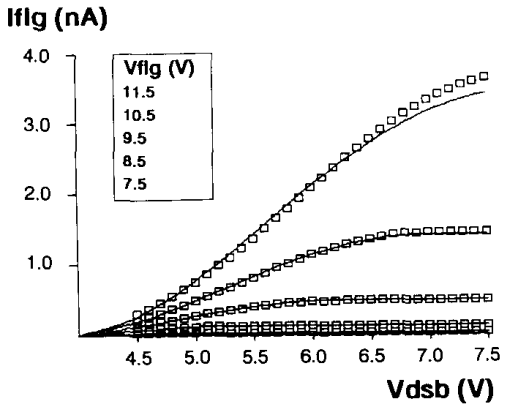

(b)

Fig. 13. Measured (squares) and fitted (solid lines) injector (a) and "floating" gate current (b) of device D, using the model for SPICE. The RMS error is $2.9 \%$.

after measurement, whereas the solid lines denote the theoretical curves according to the model that has been explained in Section IV. The parameters used in this model have been extracted by the parameter extraction program PROMEA [32] and are listed in Table II. The rms error of the fit is $2.9 \%$. As can be seen from Fig. 13(a), the injector current can quite well be described by the model, which is composed of the punchthrough, and SPICE level3 MOS model. The injection probability, being modeled by a linear relationship between the gate voltage and injector current, gives a good prediction for the floating gate current (Fig. 13(b)). The extracted parameters (Table II) do have realistic physical values. The relatively high value, found for the bulk factor gamma, originates from the implantation which should prevent the trunk from the buried injector to the inversion channel [27]. Also the injector resistance $R_{\text {inj }}$ of $1.4 \mathrm{k} \Omega$ is in good agreement with the value that was expected from the layout configuration.

It is not the intention of this paper to check or approve the physical correctness of the LEM. However, it is interesting to examine, whether the experimental results concerning the reported LEM parameters [6], [13] are applicable to our devices. Hence, the injection probability is measured as a function of $V_{\mathrm{fg}}$. The injection probability $P_{i n j}$ is obtained from the division of the measured "floating" gate current by the injector current $V_{\mathrm{dsb}}$ is kept at $V_{\mathrm{tr}}$. In the $V_{\mathrm{dsb}}$ range, $V_{\mathrm{pt}}$ to $V_{\mathrm{tr}}$, the injector current hardly changes with $V_{\mathrm{fg}}$ as demonstrated in Fig. 9. So a constant potential along the whole inversion channel can be expected. The results are illustrated in Fig. 14. The squares are the measured data and the solid lines are the theoretical curves

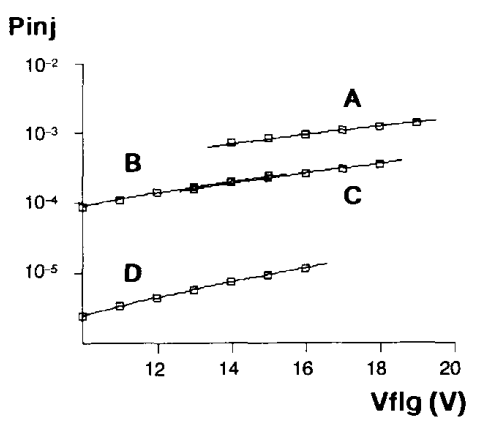

Fig. 14. The measured (squares) and fitted (solid lines) injection probability for the devices A-D using the Lucky Electron Model. The rms error is $1.6 \%$

TABLE II

The SPICE Model Parameters Giving the Best Fit with the MEasured INJECTOR and "Floating" Gate Current of Device D

\begin{tabular}{ccc}
\hline Parameter & Unit & Value \\
\hline$\phi$ & $\mathrm{V}$ & 0.64 \\
$V_{t 0}$ & $\mathrm{~V}$ & 0.56 \\
$K_{p}$ & $\mathrm{~A} \cdot \mathrm{V}^{-2}$ & $3.11 \times 10^{-5}$ \\
$\gamma$ & $\mathrm{V}^{0.5}$ & 1.54 \\
$\theta$ & $\mathrm{V}^{-1}$ & $9.63 \times 10^{-2}$ \\
$V_{\max }$ & $\mathrm{m} \cdot \mathrm{s}^{-1}$ & $6.34 \times 10^{4}$ \\
$W_{\operatorname{mos}}$ & $\mathrm{m}$ & $10 \times 10^{-6}$ \\
$L_{\mathrm{mos}}$ & $\mathrm{m}$ & $5 \times 10^{-6}$ \\
$I_{0}$ & $\mathrm{~A}$ & $1.91 \times 10^{-11}$ \\
$x_{\mathrm{pt}}$ & $\mathrm{m}$ & $1.74 \times 10^{-7}$ \\
$L_{\mathrm{pt}}$ & $\mathrm{m}$ & $0.50 \times 10^{-6}$ \\
$V_{\mathrm{pt}}$ & $\mathrm{V}$ & 2.73 \\
$\phi_{j 0}$ & $\mathrm{~V}$ & 0.58 \\
$R_{\mathrm{inj}}$ & $\Omega$ & $1.44 \times 10^{3}$ \\
$P_{0}$ & $\cdot$ & $2.01 \times 10^{-6}$ \\
$P_{k}$ & $\mathrm{~A}$ & $1.09 \times 10^{-2}$ \\
$V_{P 0}$ & $\mathrm{~V}$ & 7.5 \\
\hline
\end{tabular}

according to the LEM. Equation (7) is used to calculate the distance $d$ (this equation is valid for $\phi_{\mathrm{ch}} \leq \phi_{\mathrm{dep}}$ ). The minor change in the position as well as the height of the potential barrier $\phi_{f}$ for $V_{\mathrm{dsb}}=V_{\mathrm{tr}}$ only introduces a small error. A value of $9.2 \mathrm{~nm}$ was used for $\lambda$ [6], [13]. The image force barrier lowering constant $\beta$ was taken as $2.59 \times 10^{-4}(\mathrm{~V} \cdot \mathrm{cm})^{1 / 2}$. The measured data have been fitted with the LEM by means of the doping concentration $N_{a}$ and the empirical LEM parameters $A$ and $\alpha$. The theoretical curves correspond very well to the measured data. The rms error is $1.6 \%$.

The assumption of a homogeneously doped substrate above the injector does not disturb the validity of the LEM. In our devices the doping profile in the area above the injector is rather constant and does not have a pronounced shape. Our derivation concerning the injection probability may not be appropriate for devices with a high surface concentration declining to the injector, because an increase of the electrical field in the vicinity of the $\mathrm{Si}-\mathrm{SiO}_{2}$ interface will greatly enhance the injection probability.

The parameters of the theoretical injection model, which give the best fit in measured injection probability, are tabulated in Table III. The value of $A$ is 2.9 and it corresponds very well to the value which has been reported by Ning et al. [13]. The 
TABLE III

The Measured Punchthrough Voltage $V_{\mathrm{pt}}$ and the Nonideality factor $n$ of Devices A-D (The fitted doping concentration $N_{a}$ as well as the parameters of the Lucky Electron Model are included.)

\begin{tabular}{ccccclc}
\hline Device & $V_{\mathrm{pt}}(\mathrm{V})$ & $V_{\mathrm{tr}}(\mathrm{V})$ & $n$ & $N_{a}\left(\mathrm{~cm}^{-3}\right)$ & & \\
\hline A & 6.85 & 8.0 & 3.6 & $5.27 \times 10^{16}$ & $\lambda(\mathrm{nm})$ & 9.2 \\
B & 4.75 & 5.8 & 3.2 & $4.69 \times 10^{16}$ & $\alpha\left(\mathrm{V}^{1 / 3} \cdot \mathrm{cm}^{2 / 3}\right)$ & $7.52 \times 10^{-6}$ \\
C & 5.35 & 6.5 & 3.6 & $5.33 \times 10^{16}$ & $\beta\left((\mathrm{V} \cdot \mathrm{cm})^{1 / 2}\right)$ & $2.59 \times 10^{-4}$ \\
D & 2.75 & 3.8 & 3.0 & $4.36 \times 10^{16}$ & $A$ & 2.9 \\
\hline
\end{tabular}

"tunneling barrier lowering" constant $\alpha$ of our devices, $7.5 \times$ $10^{-6} \mathrm{~V}^{1 / 3} \cdot \mathrm{cm}^{2 / 3}$, is slightly less than the $1 \times 10^{-5}$, determined by Ning et al. [13]. However, the value is in contrast with the $4 \times 10^{-5}$, obtained by Tam et al. [6]. From these results it can be stated that the LEM can be used in our devices to predict the injection probability in the case of a constant surface potential.

It can be seen from Fig. 14 and Table III that the doping profile in the injector area strongly influences the punchthrough voltage and injection probability. Therefore, a reproducible VIPMOS structure can only be realized by well-controlled processing, such as ion implantation.

The highest injection probabilities are acquired by device $\mathrm{A}$. This directly results from the high punchthrough voltage. On the other hand, the injection probabilities as obtained from device $\mathrm{B}$ and $\mathrm{C}$ are nearly the same, although the punchthrough voltages differ $0.6 \mathrm{~V}$. This can be explained as follows: the nonideality factor $n$ of device $\mathrm{C}$ is greater than that of device $\mathrm{B}$; thus the position of the potential barrier $\phi_{f}$ is shifted towards the $\mathrm{Si}-\mathrm{SiO}_{2}$ interface surface in the latter case, thereby compensating the effect of the lower value of the punchthrough voltage.

The dependence of the injection probability on the electrical field in the gate oxide is more pronounced for the devices with a low punchthrough voltage. It is evident that the use of image force barrier lowering and "tunneling barrier lowering" are of great importance for 5-V-only circuits applying VIPMOS devices, in order to increase the injection probability.

\section{CONCLUSIONS}

A novel high-energy ion-implanted VIPMOS structure has been presented. The structure includes a local buried injector, which is used to supply the electrons for the SHE injection. The fabrication of the VIPMOS structure involves a local overlap of the wells of a retrograde twin-well CMOS process and one extra implantation to obtain an accurate Gummel number of the substrate area above the injector. One extra mask is necessary to cover the trunk, that shows up from the buried injector to the $\mathrm{Si}-\mathrm{SiO}_{2}$ interface due to mask edge effects. For this reason, the VIPMOS EPROM cell consumes more area than an EPROM cell using CHE injection. The injector is activated by means of punchthrough. This makes the structure suitable for VLSI applications without the danger of latchup. The punchthrough voltage can be set by the doping profile of the p-well. The local buried injector offers new possibilities for programming EPROM's using SHE injection.

A high Gummel number of the area above the injector results in a high punchthrough voltage and a high injection probability (about $1 \times 10^{-3}$ ). In consequence of the high injection probability and effective injection mechanism, high oxide current densities of $1 \mathrm{~A} \cdot \mathrm{cm}^{-2}$ and programming times of $10 \mu \mathrm{s}$ have been measured. On the other hand, the punchthrough voltage can be adjusted to a low value of about $3 \mathrm{~V}$, which enables the application of the VIPMOS structure in EPROM's for analog circuitry or 5 -V-only digital memories. For this purpose, a model was proposed which has been implemented in SPICE. This model can very well predict the injector and floating gate current.

The parameters of the LEM, obtained from a parameter extraction, are in good agreement with the parameters as reported by Ning et al. [13]. Only the dependence of the $\mathrm{Si}-\mathrm{SiO}_{2}$ potential barrier on the "tunneling lowering"' term is slightly less

\section{ACKNOWLEDGMENT}

The authors wish to thank G. Boom and A. Kooy for processing the devices. The stimulating discussions with L. Warmerdam and P. Ragay are greatly appreciated.

\section{REFERENCES}

[1] Y. Iwata, M. Momodomi, T. Tanaka, H. Oodaira, Y. Itoh, R. Nakayama, R. Kirisawa, S. Aritome, T. Endoh, R. Shirota, K. Ohuchi, and F. Masuoka, "A high-density NAND EEPROM with block-page programming for microcomputer applications,' IEEE J. Solid-State Circuits, vol. 25, pp. 417-424, 1990

[2] E. Säckinger, and W. Guggenbühl, "An analog trimming circuit based on a floating-gate device," IEEE J. Solid-State Circuits, vol. 23, pp. 1437-1440, 1988.

[3] M. H. White, I. A. Mack, G. M. Borsuk, D. R. Lampe, and F. J. Kub, "Charge-coupled device (CCD) adaptive discrete analog signal processing," IEEE J. Solid-State Circuits, vol. SC-14, pp. 132-146, 1979

[4] K. Goser, "Technological challenge of artificial neural networks," in Proc. ESSDERC, pp. 321-328, 1989.

[5] G. J. Hemink, "Design and evaluation of an SHE injection 16k flash-E ${ }^{2}$ PROM using a local buried injector," to be published.

[6] S. Tam, P. K. Ko, and C. Hu, "Lucky-electron model of channel hot-electron injection in MOSFET's,' IEEE Trans. Electron Devices, vol. ED-31, pp. 1116-1125, 1984

[7] A. T. Wu, T. Y. Chan, P. K. Ko, and C. Hu, "A novel highspeed, 5-volt programming EPROM structure with source-side injection," in IEDM Tech. Dig., pp. 584-587, 1986.

[8] C. Hu, S. C. Tam, F. C. Hsu, P. K. Ko, T. Y. Chan, and K. W. Terrill, "Hot-electron-induced MOSFET degradation-Model, monitor, and improvement,' IEEE Trans. Electron Devices, vol ED-32, pp. 375-385, 1985.

[9] K. Yoshikawa, S. Mori, Y. Kaneko, Y. Ohshima, N. Arai, and E. Sakagami, "0.6 $\mu \mathrm{m}$ EPROM cell design based on a new scaling scenario," in IEDM Tech. Dig., pp. 587-590, 1989.

[10] D. R. Wolters, "Growth, conduction, trapping and breakdown of $\mathrm{SiO}_{2}$ layers on silicon," Ph.D. dissertation, University of Groningen, The Netherlands, 1985.

[11] J. F. Verwey, "Nonavalanche injection of hot carriers into $\mathrm{SiO}_{2}$," J. Appl. Phys., vol. 44, pp. 2681-2687, 1973.

[12] J. F. Verwey, R. P. Kramer, and B. J. de Maagt, "Mean free path of hot electrons from silicon to silicon dioxide," $J$. Appl. Phys., vol. 42, pp. 2612-2619, 1971 
[13] T. H. Ning, C. M. Osburn, and H. N. Yu "Emission probability of hot electrons from silicon into silicon dioxide," J. Appl. Phys., vol. 48 , pp. $286-293,1977$.

[14] J. S. Witters, G. Groeseneken, and H. E. Maes, "Analysis and modeling of on-chip high voltage generator circuits for use in EEPROM circuits," IEEE J. Solid-State Circuits, vol. 24, pp. 1372-1380, 1989.

[15] J. J. Sanchez, K. K. Hsueh, and T. A. DeMassa, "Drain-engineered hot-electron-resistant device structures: A review," IEEE Trans. Electron Devices, vol, 36, pp. 1125-1132, 1989.

[16] J. F. Verwey, and R. P. Kramer, "Atmos-An electrically reprogrammable read-only memory device," IEEE Trans. Electron Devices, vol. ED-21, pp. 631-636, 1974.

[17] B. Eitan, J. L. McCreary, D. Amrany, and J. Shappir, "Substrate hot-electron EPROM," IEEE Trans. Electron Devices, vol. ED-31, pp. 934-942, 1984.

[18] J. L. McCreary, B. Eitan, and D. Amrany, "Techniques for a 5-V-only 64K EPROM based upon substrate hot-electron injection," IEEE J. Solid-State Circuits, vol. SC-19, pp. 135-143, 1984

[19] R. C. M. Wijburg, G. J. Hemink, L. Praamsma, and J. Middelhoek, "VIPMOS, a local buried injector for EPROMs," in Proc. ESSDERC, pp. 915-918, 1989

[20] J. Lohstroh and A. Slob, "Punch-through cell for dense bipolar ROMs," in Proc. Solid-State Conf., pp. 20-21, 1978.

[21] T. Mouthaan, and M. Vertregt, "A vertically integrated dynamic RAM-cell: Buried bit line memory cell with floating transfer line," Solid-State Electron., vol. 29, pp. 1289-1294, 1986

[22] J. Lohstroh, J. J. M. Koomen, A. T. van Zanten, and R. H. W. Salters, "Punch-through currents in $\mathrm{P}^{+} \mathrm{NP}^{+}$and $\mathrm{N}^{+} \mathrm{PN}^{+}$sandwich structures-I," Solid-State Electron., vol. 24, pp. 805-814, 1981

[23] - "Punch-through currents in $\mathrm{P}^{+} \mathrm{NP}^{+}$and $\mathrm{N}^{+} \mathrm{PN}^{+}$sandwich structures-II," Solid-State Electron., vol. 24, pp. 815-820, 1981.

[24] W. Shockley, "Problems related to p-n junctions in silicon," Solid-State Electron., vol. 2, pp. 35-67, 1961

[25] A. Stolmeijer, "A twin-well CMOS process using high energy ion implantation," IEEE Trans. Electron Devices, vol. ED-33, pp. $450-457,1986$

[26] C. P. Ho, P. D. Plummer, S. E. Hansen, and R. W. Dutton, "VLSI process modeling-SUPREM III," IEEE Trans. Electron Devices, vol. ED-30, pp. 1438-1453, 1983.

[27] R. C. M. Wijburg, G. J. Hemink, and J. Middelhoek, "'Strongly asymmetric doping profiles at mask edges in high energy ion implantation," IEEE Trans. Electron Devices, vol. 37, pp. 79-87, 1990

[28] G. J. Hemink, R. C. M. Wijburg, R. Cuppens, and J. Middelhoek, "High efficiency hot electron injection for EEPROM applications using a buried injector," in Proc. SSDM, pp. 133-136, 1989 .

[29] E. van Schie, Ph. B. M. Wolbert, and J. Middlehoek, "TRENDY: A 2D process and device modeling and simulation environment," in Software Tools for Process, Device and Circuit Modeling, NASECODE VI CONF., pp. 155-161, 1989.

[30] E. Sun, J. Moll, J. Berger, and B. Adlers, "Breakdown mechanism in short-channel MOS transistors," in IEDM Tech. Dig. pp. $478,482,1978$

[31] P. Antognetti and G. Massobrio, Semiconductor Device Modeling with SPICE. New York, NY: McGraw-Hill, 1987.

[32] Ph. B. M. Wolbert, R. C. M. Wijburg, E. van Schie, and P. F. A. Middelhoek, "PROMEA: A flexible window-based parameter extraction program for PC's," in Software Tools for Process, Device and Circuit Modeling, NASECODE VI Conf., pp. 162-167, 1989

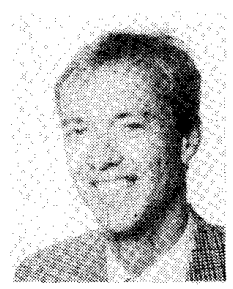

Rutger C. Wijburg was born in Nijmegen, The Netherlands, in 1962. He received the M.Sc. degree in electrical engineering from the University of Twente, Enschede, The Netherlands, in 1986. Currently, he is working toward the Ph.D. degree at the University of Twente.

His research has been concentrated on the design of SRAM's. In 1985, he joined Siemens, Munich, Germany, where he was engaged in the development of chip cards. His research topics include combined bipolar-CMOS processing using highenergy ion implantation, the application of BiCMOS circuits, the development and application of new EEPROM structures, and device modeling.

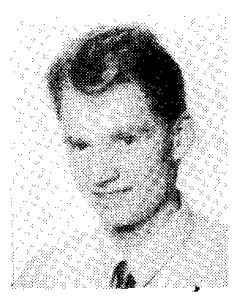

Gertjan J. Hemink (S'88) was born in Wisch, The Netherlands, on December 7, 1964. He received the M.Sc. degree in electrical engineering from the University of Twente, Enschede, The Netherlands, in 1988. Since March 1988 , he has been working towards the Ph.D. degree at the University of Twente.

At present his main research interests are the development and application of new EEPROM structures using high-energy ion implantation, the design of Flash EEPROM's, and the development of an EEPROM cell for analog applications. His general interests are in the field of device development and application.

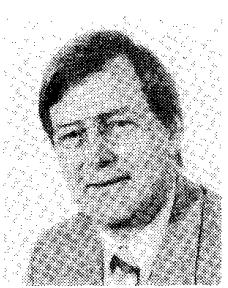

Jan Middelhoek, deceased, was born in Rotterdam, The Netherlands, in 1935. He received the M.Sc. degree from the Free University, Amsterdam, The Netherlands, and the D.Sc. degree from the State University, Leiden, The Netherlands.

He was a professor at the University of Twente, The Netherlands, where he taught IC technology and electronics. He was the head of the Laboratory for Integrated Circuits. His research was concentrated on the application of megaelectronvolt ion implantation in IC fabrication. He was a member of the German/Dutch MEGA expert committee for the development of submicrometer-technology and chairman of the Semiconductors' group of the Foundation of Fundamental Research on Matter (FOM), Utrecht. He was also a coordinator of a student mobility program in the field of microelectronics in the framework of Euramusprogram of the E.E.C.

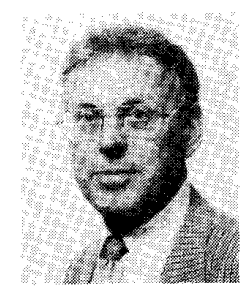

Hans Wallinga (M'81) received the M.Sc. degree in physics from the State University of Utrecht, The Netherlands, and the Ph.D. degree in technical sciences from the University of Twente, Enschede, The Netherlands.

He joined the University of Twente in 1967 where he initially was involved in device physics and device characterization of MOST devices and CCD's. His research activities gradually also included the design and testing of sampled data and adaptive signal processing circuits. He is now professor in semiconductor devices and heading the sub-department on IC Technology and Electronics in the faculty of Electrical Engineering, University of Twente.

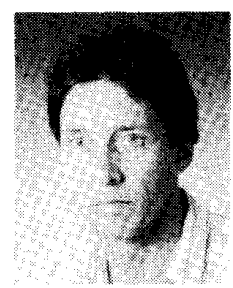

Ton J. Mouthaan (M'89) received the B.Sc. degree in electrical engineering in 1973, and the M.Sc. degree in 1977, both from the University of Twente, Einschade, The Netherlands. In 1986, he received the Ph.D. degree from the same university.

After a short period as Research Assistant at the University of Twente, he went as a Lecturer to the University of Zambia to establish a Department of Electronics there. His Ph.D. research (at the University of Twente) delt with high-energy ion implantation for the fabrication of vertical devices. After two more years at the University of Zambia, as Senior Lecturer, he returned to the University of Twente as Associate Professor heading research groups in modeling and simulation and CAD for the synthesis of analog circuits. He also lectures on IC technology. 